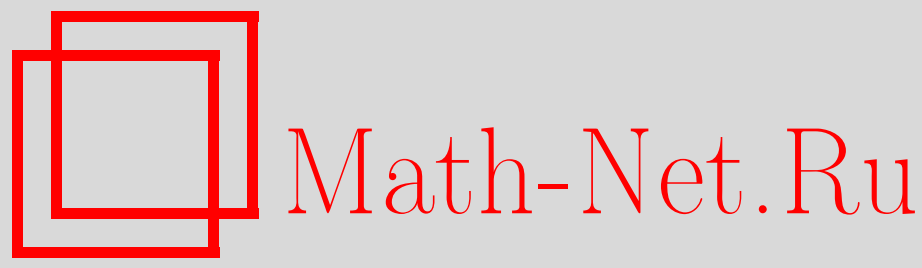

М. С. Брузон, Явные решения обобщенного уравнения Буссинеска, ТМФ, 2009, том 160, номер 1, 23-34

DOI: https://doi.org/10.4213/tmf6375

Использование Общероссийского математического портала Math-Net.Ru подразумевает, что вы прочитали и согласны с пользовательским соглашением http: //www.mathnet.ru/rus/agreement

Параметры загрузки:

IP: 35.173 .219 .149

26 апреля 2023 г., 12:56:21

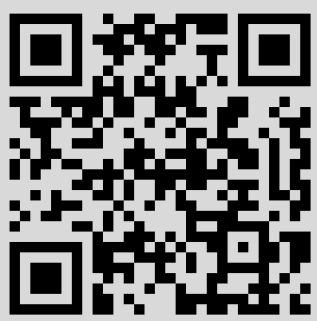




\title{
ФИЗИКА
}

Том 160, № 1

июль, 2009

\section{ЯВНЫЕ РЕШЕНИЯ ОБОБЩЕННОГО УРАВНЕНИЯ БУССИНЕСКА}

\begin{abstract}
$\mathrm{C}$ помощью теории симметрийных редукций для уравнений в частных производных проведен анализ обобщенного уравнения Буссинеска. Анализ с использованием группы симметрий Ли для этого уравнения показывает, что оно допускает только двухпараметрическую группу точечных симметрий, отвечающую решениям в виде уединенной движущейся волны. Для построения точных решений применяются две процедуры: прямой метод и метод $\frac{G^{\prime}}{G}$-разложения. Решения в виде бегущих волн выражены в терминах гиперболических, тригонометрических и рациональных функций.
\end{abstract}

Ключевые слова: уравнение в частных производных, симметрия, решение.

\section{1. ВВЕДЕНИЕ}

Уравнение Буссинеска принадлежит к семейству уравнений Кортевега-де Фриза и описывает движения под действием гравитации длинных волн на мелкой воде, распространяющихся в обоих направлениях. Это уравнение имеет вид

$$
u_{t t}=u_{x x}+c u_{x x x x}+\left(u^{2}\right)_{x x} .
$$

Здесь $u=u(x, t)$ - дифференцируемая вплоть до нужного порядка функция. При $c=-1$ мы имеем корректно поставленное Буссинеска, а при $c=1$ - некорректно поставленное классическое уравнение [1], [2]. С другой стороны, мы имеем поправленное уравнение Буссинеска

$$
u_{t t}=u_{x x}+u_{x x t t}+\left(u^{2}\right)_{x x} .
$$

В физических исследованиях распространения нелинейных волн в волноводах необходимо учитывать взаимодействие волноводов с внешней средой и, следовательно, возможность обмена энергией через внешние поверхности волноводов. При учете обмена энергией между стержнем и средой необходимо принимать в расчет

* Departamento de Matemáticas, Universidad de Cádiz, Cádiz, Spain. E-mail: matematicas.casem@uca.es 
диссипацию деформационной волны в вязкой внешней среде. Уравнение с двумя дисперсиями, получаемое из принципа Гамильтона, имеет третий порядок:

$$
u_{t t}-u_{x x}=\frac{1}{4}\left(c u^{3}+6 u^{2}+a u_{t t}-b u_{x x}+d u_{t}\right)_{x x}
$$

где $a, b, c$ и $d$ - некоторые положительные числа. Это уравнение исследовалось в работах [3], [4]. В работах [5]-[8] рассматривалась начально-краевая задача и задача Коши для обобщенного уравнения с двумя дисперсиями, которое включает уравнение (3) как частный случай.

Симметрийные редукции и точные решения имеют немало различных важных приложений в контексте дифференциальных уравнений. Поскольку решения уравнений в частных производных (УЧП) асимптотически стремятся к решениям уравнений низших размерностей, получаемых с помощью симметрийных редукций, некоторые из этих специальных решений иллюстрируют важные физические явления. В частности, точные решения, полученные на основе симметрийных методов, могут быть зачастую эффективно использованы при исследовании таких свойств, как асимптотические поведения и "раздутия". Более того, явные решения (например, решения, найденные с помощью симметрийных методов) могут играть важную роль в конструировании и проверке численных интеграторов; с помощью этих решений можно на практике проводить важные проверки точности и надежности таких интеграторов [9]. В работах [9]-[13] исследовались симметрийные редукции и точные решения некоторого уравнения Буссинеска. В работах [14]-[18] были применены метод групп Ли и неклассический метод для вывода симметрий некоторых уравнений Буссинеска.

В настоящей работе рассматривается обобщенное уравнение Буссинеска

$$
\Delta \equiv u_{t t}-u_{x x t t}+u_{x x x x t t}+c u_{x x x x}-u_{x x}=(f(u))_{x x}
$$

и при $c \neq 0$ исследуется, для каких функций $f(u)$ это уравнение обладает классической группой симметрий. Необходимые факты теории и описание метода содержатся в работах [19], [20]. С применением симметрийных редукций исходное нелинейное УЧП сводится к нелинейному обыкновенному дифференциальному уравнению (ОДУ). Точные решения затем получаются из этого уравнения применением прямого метода. Используя метод $\frac{G^{\prime}}{G}$-разложения, который был недавно введен Вангом с соавторами в работе [21], мы получаем новые точные решения уравнения (4) для $f(u)=u^{2}$.

\section{2. КЛАССИЧЕСКИЕ СИММЕТРИИ}

Для того чтобы применить классический метод Ли к уравнению (4), рассмотрим однопараметрическую группу Ли инфинитезимальных преобразований в пространстве переменных $x, t, u$, задаваемую формулами

$$
\begin{aligned}
x^{*} & =x+\epsilon \xi(x, t, u)+O\left(\epsilon^{2}\right), \\
t^{*} & =t+\epsilon \tau(x, t, u)+O\left(\epsilon^{2}\right), \\
u^{*} & =u+\epsilon \eta(x, t, u)+O\left(\epsilon^{2}\right),
\end{aligned}
$$


в которых $\epsilon$ представляет собой параметр группового преобразования. Потребуем, чтобы это преобразование оставляло инвариантным множество решений уравнения (4). Это требование приводит к переопределенной системе линейных уравнений на малые величины $\xi(x, t, u), \tau(x, t, u)$ и $\eta(x, t, u)$. Связанная с ними алгебра инфинитезимальных преобразований симметрии есть набор векторных полей вида

$$
\mathbf{v}=\xi(x, t, u) \partial_{x}+\tau(x, t, u) \partial_{t}+\eta(x, t, u) \partial_{u}
$$

Функции $u=u(x, t)$, инвариантные относительно инфинитезимальных преобразований $\mathbf{v}$, представляют собой по существу решения уравнения, возникающего как "условие инвариантной поверхности"

$$
\eta(x, t, u)-\xi(x, t, u) u_{x}-\tau(x, t, u) u_{t}=0 .
$$

Переменные симметрии можно найти, разрешая это условие инвариантной поверхности. При редукции УЧП превращаются в ОДУ.

Перейдем к классическому анализу группы симметрий Ли для уравнения (4). Множество решений этого уравнения является инвариантным относительно преобразований (5), если $\operatorname{pr}^{(6)} \mathbf{v}(\Delta)=0$ при $\Delta=0$, где $\operatorname{pr}^{(6)} \mathbf{v}-$ продолжение шестого порядка для векторного поля (6). При этом возникают шестнадцать определяющих уравнений для малых величин. Из этой системы следует, что если $f$ представляет собой произвольную функцию, то единственными симметриями, которые допускает уравнение (4), будут симметрии

$$
\xi=\lambda, \quad \tau=\mu, \quad \eta=0,
$$

задаваемые группой пространственно-временных преобразований $\mathbf{v}_{1}=\partial_{x}, \mathbf{v}_{2}=\partial_{t}$. Подставив (8) в условие инвариантной поверхности (7), получим подобную переменную и подобное решение:

$$
z=\mu x-\lambda t, \quad u(x, t)=h(z) .
$$

После подстановки этих соотношений в (4) имеем

$$
\lambda^{2} \mu^{4} h^{\prime \prime \prime \prime \prime \prime \prime}+\mu^{2}\left(c \mu^{2}-\lambda^{2}\right) h^{\prime \prime \prime \prime}+\left(\lambda^{2}-\mu^{2}\right) h^{\prime \prime \prime}-\mu^{2} f_{h} h^{\prime \prime}-\mu^{2} f^{\prime \prime}\left(h^{\prime}\right)^{2}=0 .
$$

Дважды интегрируя это уравнение по переменной $z$, получим

$$
\lambda^{2} \mu^{4} h^{\prime \prime \prime \prime}+\mu^{2}\left(\mu^{2} c-\lambda^{2}\right) h^{\prime \prime}+\left(\lambda^{2}-\mu^{2}\right) h-\mu^{2} f(h)+A z+B=0,
$$

где $A$ и $B$ - константы интегрирования.

При $f(u)=a u+b$ уравнение (4) допускает дополнительные симметрии и становится линейным УЧП, поэтому мы не рассматриваем этот случай.

\section{3. РЕШЕНИЯ ТИПА БЕГУЩЕЙ ВОЛНЫ}

В этом разделе мы получим решения в виде бегущей волны для уравнения (11), применяя две процедуры: прямой метод и метод $\frac{G^{\prime}}{G}$-разложения. 
3.1. Прямой метод. Рассмотрим уравнение (11) с $A=B=0$ :

$$
h^{\prime \prime \prime \prime}+\frac{\mu^{2} c-\lambda^{2}}{\mu^{2} \lambda^{2}} h^{\prime \prime}+\frac{\lambda^{2}-\mu^{2}}{\mu^{4} \lambda^{2}} h-\frac{1}{\mu^{2} \lambda^{2}} f(h)=0 .
$$

Это уравнение можно переписать в виде

$$
h^{\prime \prime \prime \prime}+b h^{\prime \prime}+F(h)=0,
$$

где $b=\left(\mu^{2} c-\lambda^{2}\right) / \mu^{2} \lambda^{2}$ и

$$
F(h)=\frac{\lambda^{2}-\mu^{2}}{\mu^{4} \lambda^{2}} h-\frac{1}{\mu^{2} \lambda^{2}} f(h) .
$$

Уравнение (13) допускает решения вида

$$
h=\alpha H^{\beta}(z),
$$

где $\alpha$ и $\beta$ - параметры, а $H(z)$ - решение уравнения Якоби [22]

$$
\left(H^{\prime}\right)^{2}=r+p H^{2}+q H^{4}
$$

с постоянными $r, p$ и $q$. Подставив (15) в (13) и применив тождества для стандартных эллиптических функций Якоби, получим уравнение в терминах $h$ и $F(h)$. Так как эти уравнения весьма громоздки, здесь мы их не приводим, но читатель может их найти в работе [23]. Из этих уравнений получаются функции $F(h)$, в которых $h$ - решение уравнения (13).

СлУчАЙ 1. Если $H(z)=\operatorname{sn} z$, то $h(z)=\alpha \operatorname{sn}^{\beta} z$ и

$$
F(h)=\alpha_{1} h^{1+4 / \beta}+\alpha_{2} h^{1+2 / \beta}+\alpha_{3} h^{1-4 / \beta}+\alpha_{4} h^{1-2 / \beta}+\alpha_{5} h,
$$

где

$$
\begin{aligned}
\alpha_{1}= & -\alpha^{-4 / \beta}\left[\beta^{4} m^{4}+\left(6 \beta^{3}+8 \beta^{2}+4 \beta\right) m^{3}+\left(3 \beta^{2}+2 \beta\right) m^{2}\right], \\
\alpha_{2}= & \alpha^{-2 / \beta}\left[\left(2 \beta^{4}-6 \beta^{3}+8 \beta^{2}-4 \beta\right) m^{4}+\left(12 \beta^{3}-6 \beta^{2}+8 \beta\right) m^{3}+\right. \\
& \left.+\left(2 \beta^{4}+(6-b) \beta^{2}\right) m^{2}+\left(6 \beta^{3}+8 \beta^{2}+(4-b) \beta\right) m\right], \\
\alpha_{3}= & -\alpha^{4 / \beta} \beta^{4}+6 \alpha^{4 / \beta} \beta^{3}-11 \alpha^{4 / \beta} \beta^{2}+6 \alpha^{4 / \beta} \beta, \\
\alpha_{4}= & \left(2 \alpha^{2 / \beta} \beta^{4}-12 \alpha^{2 / \beta} \beta^{3}+22 \alpha^{2 / \beta} \beta^{2}-12 \alpha^{2 / \beta} \beta\right) m^{2}+ \\
& +\left(\alpha^{2 / \beta} b-4 \alpha^{2 / \beta}\right) \beta+2 \alpha^{2 / \beta} \beta^{4}-6 \alpha^{2 / \beta} \beta^{3}+\left(8 \alpha^{2 / \beta}-\alpha^{2 / \beta} b\right) \beta^{2}+ \\
& +\left(6 \alpha^{2 / \beta} \beta^{3}-14 \alpha^{2 / \beta} \beta^{2}+8 \alpha^{2 / \beta} \beta\right) m, \\
\alpha_{5}= & \left(-\beta^{4}+6 \beta^{3}-11 \beta^{2}+6 \beta\right) m^{4}+\left(-6 \beta^{3}+14 \beta^{2}-8 \beta\right) m^{3}+ \\
& +\left(-4 \beta^{4}+12 \beta^{3}+(b-19) \beta^{2}+(10-b) \beta\right) m^{2}+ \\
& +\left(-12 \beta^{3}+6 \beta^{2}+(b-8) \beta\right) m-\beta^{4}+b \beta^{2} .
\end{aligned}
$$


СлУчАЙ 2. Если $H(z)=\operatorname{cn} z$, то $h(z)=\alpha \mathrm{cn}^{\beta} z$ и

$$
F(h)=\beta_{1} h^{1+4 / \beta}+\beta_{2} h^{1+2 / \beta}+\beta_{3} h^{1-4 / \beta}+\beta_{4} h^{1-2 / \beta}+\beta_{5} h,
$$

где

$$
\begin{aligned}
\beta_{1}= & \alpha^{-4 / \beta} \beta m^{2}\left(\beta^{3} m^{2}+6 \beta^{2} m+8 \beta m+4 m+3 \beta+2\right), \\
\beta_{2}= & -\alpha^{-2 / \beta} \beta m\left(4 \beta^{3} m^{3}-6 \beta^{2} m^{3}+8 \beta m^{3}-4 m^{3}+18 \beta^{2} m^{2}+b+\right. \\
& \left.+2 \beta m^{2}+12 m^{2}-2 \beta^{3} m+b \beta m+6 \beta m-6 \beta^{2}-8 \beta-4\right), \\
\beta_{3}= & \alpha^{4 / \beta}(\beta-3)(\beta-2)(\beta-1) \beta(m-1)^{2}(m+1)^{2}, \\
\beta_{4}= & -\alpha^{2 / \beta}(\beta-1) \beta(m-1)(m+1)\left(4 \beta^{2} m^{2}-14 \beta m^{2}+16 m^{2}+\right. \\
& \left.+6 \beta m-8 m-2 \beta^{2}+4 \beta+b-4\right), \\
\beta_{5}= & \beta\left(6 \beta^{3} m^{4}-18 \beta^{2} m^{4}+27 \beta m^{4}-14 m^{4}+18 \beta^{2} m^{3}-20 \beta m^{3}+\right. \\
& +16 m^{3}-6 \beta^{3} m^{2}+12 \beta^{2} m^{2}+2 b \beta m^{2}-13 \beta m^{2}-b m^{2}+6 m^{2}- \\
& \left.-12 \beta^{2} m+6 \beta m+b m-8 m+\beta^{3}-b \beta\right) .
\end{aligned}
$$

СлУчАй 3. Если $H(z)=\operatorname{dn} z$, то $h(z)=\alpha \operatorname{dn}^{\beta} z$ и

$$
F(h)=\gamma_{1} h^{1-2 / q}+\gamma_{2} h+\gamma_{3} h^{1+2 / q}+\gamma_{4} h^{1-4 / q}+\gamma_{5} h^{1+4 / q},
$$

где

$$
\begin{aligned}
\gamma_{1}= & -\alpha^{-4 / \beta} m^{-4} \beta\left(8 m^{3}+28 \beta m^{2}-12 m^{2}+12 \beta^{2} m-28 \beta m+\right. \\
& \left.+16 m+\beta^{3}-6 \beta^{2}+11 \beta-6\right), \\
\gamma_{2}= & -\alpha^{-2 / \beta} m^{-4} \beta\left(4 m^{5}+28 \beta m^{4}-12 m^{4}+18 \beta^{2} m^{3}-42 \beta m^{3}-\right. \\
& -2 b m^{3}+16 m^{3}+2 \beta^{3} m^{2}-12 \beta^{2} m^{2}-b \beta m^{2}-34 \beta m^{2}+b m^{2}+ \\
& \left.+12 m^{2}-36 \beta^{2} m+84 \beta m-48 m-4 \beta^{3}+24 \beta^{2}-44 \beta+24\right), \\
\gamma_{3}= & -\alpha^{4 / \beta} m^{-4}(\beta-3)(\beta-2)(\beta-1) \beta(m-1)^{2}(m+1)^{2}, \\
\gamma_{4}= & \alpha^{2 / \beta} m^{-4}(\beta-1) \beta(m-1)(m+1)\left(6 \beta m^{3}-8 m^{3}+2 \beta^{2} m^{2}-\right. \\
& \left.-10 \beta m^{2}-b m^{2}+12 m^{2}-12 \beta m+16 m-4 \beta^{2}+20 \beta-24\right), \\
\gamma_{5}= & -\beta\left(3 \beta m^{6}-2 m^{6}+6 \beta^{2} m^{5}-14 \beta m^{5}-b m^{5}+8 m^{5}+\beta^{3} m^{4}-\right. \\
& -6 \beta^{2} m^{4}-b \beta m^{4}-17 \beta m^{4}+b m^{4}+6 m^{4}-36 \beta^{2} m^{3}+84 \beta m^{3}+ \\
& +2 b m^{3}-48 m^{3}-6 \beta^{3} m^{2}+36 \beta^{2} m^{2}+2 b \beta m^{2}-38 \beta m^{2}- \\
& -2 b m^{2}+24 m^{2}+36 \beta^{2} m-84 \beta m+48 m+6 \beta^{3}-36 \beta^{2}+ \\
& +66 \beta-36) m^{-4} .
\end{aligned}
$$

Подставляя (17), (19) и (21) в (14), получим три функции $f(h)$, в которых $h-$ решение уравнения (12), для случаев 1, 2 и 3 соответственно.

СлУчАй 1. Подставим (17) в (14), в результате имеем

$$
f(h)=\alpha_{1}^{\prime} h^{1+4 / \beta}+\alpha_{2}^{\prime} h^{1+2 / \beta}+\alpha_{3}^{\prime} h^{1-4 / \beta}+\alpha_{4}^{\prime} h^{1-2 / \beta}+\alpha_{5}^{\prime} h,
$$

где $\alpha_{i}^{\prime}=-\mu^{2} \lambda^{2} \alpha_{i}, i=1, \ldots, 4, \alpha_{5}^{\prime}=-\mu^{2} \lambda^{2} \alpha_{5}+\mu^{2}\left(\lambda^{2}-\mu^{2}\right)$, а величины $\alpha_{i}$, $i=1, \ldots, 5$, задаются формулами (18). При этом решение уравнения (12) имеет 


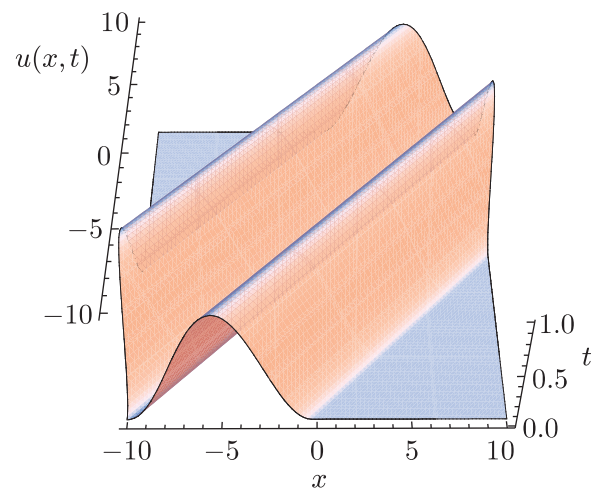

Рис. 1. Синусоподобный двойной компактон.

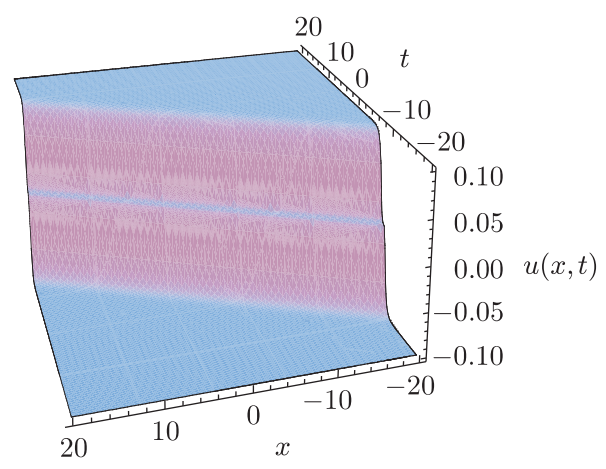

Рис. 2. Антикинк.

вид $h(z)=\alpha \operatorname{sn}^{\beta}(z \mid m)$, а точное решение уравнения (4), в котором $f(u)$ получается подстановкой $u$ вместо $h$ в формулу (23), есть

$$
u(x, t)=\alpha \operatorname{sn}^{\beta}(\mu x-\lambda t \mid m) .
$$

Далее мы приведем некоторые решения, интересные с точки зрения физики.

- При $m=0, \mu=\lambda=\sqrt{5 / 48}, \alpha=1$ и $\beta=2$ подстановка этих величин в (23) дает

$$
f(h)=-\frac{5}{288}\left(12 c^{2}-17\right)(2 h-1),
$$

и, поскольку $\operatorname{sn}(z \mid 0)=\sin z$, мы имеем частное решение

$$
u(x, t)= \begin{cases}\sin ^{2}\left[\sqrt{\frac{5}{48}}(x-t)\right], & |x-t| \leqslant \frac{2 \pi}{k}, \\ 0, & |x-t|>\frac{2 \pi}{k},\end{cases}
$$

в котором $k=\sqrt{5 / 12}$. Это решение есть синусоподобный двойной компактон, т.е. решение с двумя пиками (рис. 1).

- При $m=\mu=\beta=1, \lambda=1 / 2$ и $\alpha=1 / 4$ подстановка этих величин в (23) дает

$$
f(h)=1536 h^{5}+32 c h^{3}-168 h^{3}+\left(\frac{15}{4}-2 c\right) h,
$$

и, поскольку $\operatorname{sn}(z \mid 1)=\operatorname{th} z$, решение уравнения (11) имеет вид $h(z)=($ th $z) / 4$. При этом точное решение уравнения (4), где $f(u)$ получается при подстановке $u$ вместо $h$ в формулу (27), есть

$$
u(x, t)=\frac{1}{4} \operatorname{th}\left(x-\frac{t}{2}\right)
$$

и представляет собой решение типа кинка. 
- При $m=\mu=\alpha=1, \lambda=1 / 2$ и $\beta=3$ подстановка этих величин в (23) дает

$$
f(h)=90 h^{7 / 3}+3\left(4 c^{2}-69\right) h^{5 / 3}+\frac{3}{2}\left(4 c^{2}-21\right) h^{1 / 3}-\frac{3}{4}\left(24 c^{2}-197\right) h^{-1 / 3},
$$

и решение уравнения (11) имеет при этом вид $h(z)=\operatorname{th}^{3} z$. Соответственно, точное решение уравнения (4), в котором $f(u)$ получается при подстановке $u$ вместо $h$ в формулу (29), есть

$$
u(x, t)=\operatorname{th}^{3}\left(x-\frac{t}{2}\right)
$$

и представляет собой решение типа антикинка (рис. 2)

СлУчАЙ 2. Подставляя (19) в (14), получим

$$
f(h)=\beta_{1}^{\prime} h^{1+4 / \beta}+\beta_{2}^{\prime} h^{1+2 / \beta}+\beta_{3}^{\prime} h^{1-4 / \beta}+\beta_{4}^{\prime} h^{1-2 / \beta}+\beta_{5}^{\prime} h,
$$

где $\beta_{i}^{\prime}=-\mu^{2} \lambda^{2} \beta_{i}, i=1, \ldots, 4, \beta_{5}^{\prime}=-\mu^{2} \lambda^{2} \beta_{5}+\mu^{2}\left(\lambda^{2}-\mu^{2}\right)$, а величины $\beta_{i}$, $i=1, \ldots, 5$, задаются формулами (20). Решение уравнения (12) имеет при этом вид $h(z)=\alpha \mathrm{cn}^{\beta}(z \mid m)$. Соответственно, точное решение уравнения (4), в котором $f(u)$ получается при подстановке $u$ вместо $h$ в выражение (31), есть

$$
u(x, t)=\alpha \mathrm{cn}^{\beta}(\mu x-\lambda t \mid m) .
$$

- При $m=0, \mu=\lambda=\sqrt{5 / 48}, \alpha=1$ и $\beta=2$ подстановка этих величин в (31) дает

$$
f(h)=\frac{5}{288}\left(12 c^{2}-17\right)(2 h-1) .
$$

Поскольку $\operatorname{cn}(z \mid 0)=\cos z$, можно получить частное решение

$$
u(x, t)= \begin{cases}\cos ^{2}(\mu x-\lambda t), & |x-t| \leqslant \frac{\pi}{k}, \\ 0, & |x-t|>\frac{\pi}{k},\end{cases}
$$

где $k=\sqrt{5 / 12}$. Это решение представляет собой компактон с одним пиком.

СлУчАЙ 3. Подставив (21) в (14), получим, что

$$
f(h)=\gamma_{1}^{\prime} h^{1+4 / \beta}+\gamma_{2}^{\prime} h^{1+2 / \beta}+\gamma_{3}^{\prime} h^{1-4 / \beta}+\gamma_{4}^{\prime} h^{1-2 / \beta}+\gamma_{5}^{\prime} h,
$$

где $\gamma_{i}^{\prime}=-\mu^{2} \lambda^{2} \gamma_{i}, i=1, \ldots, 4, \gamma_{5}^{\prime}=-\mu^{2} \lambda^{2} \gamma_{5}+\mu^{2}\left(\lambda^{2}-\mu^{2}\right)$, a $\gamma_{i}, i=1, \ldots, 5$, задаются выражениями (22). Решение уравнения (12) имеет при этом вид $h(z)=\alpha \operatorname{dn}^{\beta}(z \mid m)$. Соответственно, точное решение уравнения (4), в котором $f(u)$ получается при подстановке $u$ вместо $h$ в выражение (35), есть

$$
u(x, t)=\alpha \operatorname{dn}^{\beta}(\mu x-\lambda t \mid m) .
$$

- При $m=\lambda=\mu=\alpha=1$ и $\beta=2$, подставляя эти значения параметров в (35), получим

$$
f(h)=120 h^{3}-6\left(c^{2}+19\right) h^{2}+4\left(c^{2}+3\right) h .
$$


Так как $\operatorname{dn}(z \mid 1)=\operatorname{sech} z$, можно получить частное решение $h(z)=\operatorname{sech}^{2} z$. Соответственно, точное решение уравнения (4), в котором $f(u)$ получается при подстановке $u$ вместо $h$ в выражение (37), имеет вид

$$
u(x, t)=\operatorname{sech}^{2}(x-t)
$$

и представляет собой солитон, движущийся вдоль прямой с постоянной скоростью.

3.2. Метод $\frac{G^{\prime}}{G}$-разложения. Рассмотрим обобщенное уравнение Буссинеска (4) c $f(u)=u^{2}$ и будем интересоваться его решениями в виде бегущей волны. В этом случае приведенное уравнение имеет вид

$$
\mu^{4} \lambda^{2} h^{\prime \prime \prime \prime}+\mu^{2}\left(\mu^{2} c-\lambda^{2}\right) h^{\prime \prime}+\left(\lambda^{2}-\mu^{2}\right) h-\mu^{2} h^{2}+A z+B=0 .
$$

Чтобы применить метод $\frac{G^{\prime}}{G}$-разложения к этому уравнению, предположим, что $A=0$ и что решения могут быть представлены в виде многочленов от $\frac{G^{\prime}}{G}$ :

$$
h=\sum_{i=0}^{n} a_{i}\left(\frac{G^{\prime}}{G}\right)^{i}
$$

где $a_{i}, i=0, \ldots, n,-$ некоторые константы, причем $a_{n} \neq 0$, a $G=G(z)$ удовлетворяет линейному ОДУ второго порядка

$$
G^{\prime \prime}(z)+\alpha G(z)+\beta G=0,
$$

$\alpha$ и $\beta$ - также некоторые константы, которые будут определены ниже.

Общие решения уравнения (41) имеют следующий вид: если $\alpha^{2}-4 \beta>0$, то

$$
\begin{aligned}
G(z)= & c_{1} \operatorname{ch}\left(\frac{z \alpha}{2}-\frac{1}{2} z \sqrt{\alpha^{2}-4 \beta}\right)+c_{2} \operatorname{ch}\left(\frac{\alpha z}{2}+\frac{1}{2} \sqrt{\alpha^{2}-4 \beta} z\right)- \\
& -c_{1} \operatorname{sh}\left(\frac{z \alpha}{2}-\frac{1}{2} z \sqrt{\alpha^{2}-4 \beta}\right)-c_{2} \operatorname{sh}\left(\frac{\alpha z}{2}+\frac{1}{2} \sqrt{\alpha^{2}-4 \beta} z\right)
\end{aligned}
$$

если $\alpha^{2}-4 \beta<0$, то

$$
G(z)=\left(c_{2} \cos \left(\frac{1}{2} z \sqrt{4 \beta-\alpha^{2}}\right)+c_{1} \sin \left(\frac{1}{2} z \sqrt{4 \beta-\alpha^{2}}\right)\right)\left(\operatorname{ch}\left(\frac{z \alpha}{2}\right)-\operatorname{sh}\left(\frac{z \alpha}{2}\right)\right)
$$

если $\alpha^{2}=4 \beta$, то

$$
G(z)=\left(c_{2}+c_{1} z\right)\left(\operatorname{ch}\left(\frac{z \alpha}{2}\right)-\operatorname{sh}\left(\frac{z \alpha}{2}\right)\right) .
$$

Воспользовавшись соотношениями (40) и (41), получим

$$
\begin{aligned}
h^{2} & =a_{n}^{2}\left(\frac{G^{\prime}}{G}\right)^{2 n}+\cdots, \\
h^{\prime \prime \prime \prime} & =n(n+1)(n+2)(n+3) a_{n}\left(\frac{G^{\prime}}{G}\right)^{n+4}+\cdots .
\end{aligned}
$$


Рассматривая слагаемые с $h^{\prime \prime \prime \prime}$ и $h^{2}$ в формуле (39), на основе формул (45) потребуем, чтобы $n+4=2 n$, т.е. $n=4$, и тогда выражение (40) можно записать в виде

$$
h=a_{0}+a_{1}\left(\frac{G^{\prime}}{G}\right)+a_{2}\left(\frac{G^{\prime}}{G}\right)^{2}+a_{3}\left(\frac{G^{\prime}}{G}\right)^{3}+a_{4}\left(\frac{G^{\prime}}{G}\right)^{4},
$$

причем $a_{4} \neq 0$.

Подставляя общее решение уравнения (41) в (46), получим следующее: для решения, заданного формулой (42),

$$
\begin{aligned}
h_{1}(z)= & \frac{a_{4} \alpha^{4}}{16}-\frac{a_{3} \alpha^{3}}{8}+\frac{a_{2} \alpha^{2}}{4}-\frac{a_{1} \alpha}{2}+a_{0}+ \\
& +\frac{\sqrt{\alpha^{2}-4 \beta}}{8}\left(-2 a_{4} \alpha^{3}+3 a_{3} \alpha^{2}-4 a_{2} \alpha+4 a_{1}\right) H_{1}+ \\
& +\frac{\alpha^{2}-4 \beta}{8}\left(3 a_{4} \alpha^{2}-3 a_{3} \alpha+2 a_{2}\right)\left(H_{1}\right)^{2}+ \\
& +\frac{\left(\alpha^{2}-4 \beta\right)^{3 / 2}}{8}\left(a_{3}-2 a_{4} \alpha\right)\left(H_{1}\right)^{3}+\frac{\left(\alpha^{2}-4 \beta\right)^{2}}{16} a_{4}\left(H_{1}\right)^{4}
\end{aligned}
$$

где

$$
H_{1}(z)=\frac{c_{2} \operatorname{ch}\left(\sqrt{\alpha^{2}-4 \beta} z / 2\right)+c_{1} \operatorname{sh}\left(\sqrt{\alpha^{2}-4 \beta} z / 2\right)}{c_{1} \operatorname{ch}\left(\sqrt{\alpha^{2}-4 \beta} z / 2\right)+c_{2} \operatorname{sh}\left(\sqrt{\alpha^{2}-4 \beta} z / 2\right)}
$$

для решения, заданного формулой (43),

$$
\begin{aligned}
h_{2}(z)= & \frac{a_{4} \alpha^{4}}{16}-\frac{a_{3} \alpha^{3}}{8}+\frac{a_{2} \alpha^{2}}{4}-\frac{a_{1} \alpha}{2}+a_{0}+\frac{a_{4}}{16}\left(4 \beta-\alpha^{2}\right)^{2}\left(H_{2}\right)^{4}+ \\
& +\frac{a_{3}+2 a_{4} \alpha}{8}\left(4 \beta-\alpha^{2}\right)^{3 / 2} H_{2}+\frac{3 a_{4} \alpha^{2}-3 a_{3} \alpha+2 a_{2}}{8}\left(4 \beta-\alpha^{2}\right)\left(H_{2}\right)^{2}+ \\
& +\frac{-2 a_{4} \alpha^{3}+3 a_{3} \alpha^{2}-4 a_{2} \alpha+4 a_{1}}{8} \sqrt{4 \beta-\alpha^{2}}\left(H_{2}\right)^{3},
\end{aligned}
$$

где

$$
H_{2}(z)=\frac{c_{2} \cos \left(\sqrt{4 \beta-\alpha^{2}} z / 2\right)+c_{1} \sin \left(\sqrt{4 \beta-\alpha^{2}} z / 2\right)}{c_{1} \cos \left(\sqrt{4 \beta-\alpha^{2}} z / 2\right)-c_{2} \sin \left(\sqrt{4 \beta-\alpha^{2}} z / 2\right)}
$$

для решения, заданного формулой (44),

$$
\begin{aligned}
h_{3}(z)= & \frac{a_{4} \alpha^{4}}{16}-\frac{a_{3} \alpha^{3}}{8}+\frac{a_{2} \alpha^{2}}{4}-\frac{a_{1} \alpha}{2}+a_{0}+\frac{a_{4} c_{1}^{4}}{\left(c_{1} z+c_{2}\right)^{4}}+\frac{\left(a_{3}-2 a_{4} \alpha\right) c_{1}^{3}}{\left(c_{1} z+c_{2}\right)^{3}}+ \\
& +\frac{\left(3 a_{4} \alpha^{2}-3 a_{3} \alpha+2 a_{2}\right) c_{1}^{2}}{2\left(c_{1} z+c_{2}\right)^{2}}+\frac{\left(-2 a_{4} \alpha^{3}+3 a_{3} \alpha^{2}-4 a_{2} \alpha+4 a_{1}\right) c_{1}}{4\left(c_{1} z+c_{2}\right)} .
\end{aligned}
$$

Далее мы определим $a_{i}, i=0, \ldots, 4$. Из формулы (46) найдем $h^{2}, h^{\prime \prime}$ и $h^{\prime \prime \prime \prime}$ и подставим выражения для этих величин в формулу (39). Приравнивая к нулю коэффициенты при степенях $\left(\frac{G^{\prime}}{G}\right)^{i}, i=0, \ldots, 4$, получим систему алгебраических 
уравнений для $a_{i}, \alpha, \beta, \lambda, \mu$ и $B$. Ее решения задаются следующими формулами:

$$
\begin{gathered}
a_{0}=\frac{1}{338 \lambda^{2} \mu^{2}}\left[3\left(5915 \alpha^{4} \lambda^{4}+910 c \alpha^{2} \lambda^{2}+23 c^{2}\right) \mu^{4}-\right. \\
\left.-\left(169+6\left(455 \alpha^{2} \lambda^{2}+23 c\right)\right) \mu^{2} \lambda^{2}+238 \lambda^{4}\right], \\
a_{1}=\frac{420}{13} \alpha\left[\left(13 \alpha^{2} \lambda^{2}+c\right) \mu^{2}-\lambda^{2}\right], \quad a_{2}=\frac{420}{13}\left[\left(39 \alpha^{2} \lambda^{2}+c\right) \mu^{2}-\lambda^{2}\right], \\
a_{3}=1680 \alpha \lambda^{2} \mu^{2}, \quad a_{4}=840 \lambda^{2} \mu^{2}, \\
\beta=\frac{1}{52 \lambda^{2} \mu^{2}}\left[13 \alpha^{2} \mu^{2} \lambda^{2}-\lambda^{2}+c \mu^{2}\right], \\
B=\frac{1 \quad\left[-133 \lambda^{4}-(72 c-169) \mu^{2} \lambda^{2}+36 c^{2} \mu^{4}\right]\left[205 \lambda^{4}-\right.}{114244 \lambda^{4} \mu^{2}}\left[\begin{array}{l}
\left.-(72 c+169) \mu^{2} \lambda^{2}+36 c^{2} \mu^{4}\right] .
\end{array}\right.
\end{gathered}
$$

При подстановке этих величин в (47)-(49) мы получим три типа решений вида бегущей волны для обобщенного уравнения Буссинеска (4) с $f(u)=u^{2}$.

Во-первых, решение вида

$$
\begin{aligned}
u_{1}(x, t)= & \frac{215040}{169 \lambda^{2} \mu^{2}}\left(c \mu^{2}-\lambda^{2}\right)^{2} F_{1}^{4}-\frac{6720}{169 \lambda^{2} \mu^{2}}\left(c \mu^{2}-\lambda^{2}\right)^{2} F_{1}^{2}+ \\
& +\frac{69 c^{2} \mu^{2}}{338 \lambda^{2}}-\frac{69 c}{169}+\frac{119 \lambda^{2}}{169 \mu^{2}}-\frac{1}{2}
\end{aligned}
$$

где

$$
\begin{gathered}
F_{1}=\frac{c_{2} \operatorname{ch}\left[\omega_{1}(x \mu-t \lambda)\right]+c_{1} \operatorname{sh}\left[\omega_{1}(x \mu-t \lambda)\right]}{c_{1} \operatorname{ch}\left[\omega_{1}(x \mu-t \lambda)\right]+c_{2} \operatorname{sh}\left[\omega_{1}(x \mu-t \lambda)\right]} \\
\omega_{1}=\frac{1}{2} \sqrt{\frac{1}{13}\left(-\frac{c}{\lambda^{2}}+\frac{1}{\mu^{2}}\right)} .
\end{gathered}
$$

Во-вторых, решение вида

$$
\begin{aligned}
u_{2}(x, t)= & \frac{105}{338 \lambda^{2} \mu^{2}}\left(c \mu^{2}-\lambda^{2}\right)^{2} F_{2}^{4}+\frac{105}{169 \lambda^{2} \mu^{2}}\left(c \mu^{2}-\lambda^{2}\right)^{2} F_{2}^{2}+ \\
& +\frac{69 c^{2} \mu^{2}}{338 \lambda^{2}}-\frac{69 c}{169}+\frac{119 \lambda^{2}}{169 \mu^{2}}-\frac{1}{2}
\end{aligned}
$$

где

$$
\begin{gathered}
F_{2}=-\frac{c_{2} \cos \left(\omega_{2}(x \mu-t \lambda)\right)+c_{1} \sin \left(\omega_{2}(x \mu-t \lambda)\right)}{c_{2} \sin \left(\omega_{2}(x \mu-t \lambda)\right)-c_{1} \cos \left(\omega_{2}(x \mu-t \lambda)\right)}, \\
\omega_{2}=\frac{1}{2 \sqrt{13}} \sqrt{\frac{c}{\lambda^{2}}-\frac{1}{\mu^{2}}} .
\end{gathered}
$$

Наконец, решение

$u_{3}(x, t)=\frac{840 \lambda^{2} \mu^{2} c_{1}^{4}}{\left(c_{2}-c_{1} t \lambda+c_{1} x \mu\right)^{4}}-\frac{420\left(\lambda^{2}-c \mu^{2}\right) c_{1}^{2}}{13\left(-c_{2}+c_{1} t \lambda-c_{1} x \mu\right)^{2}}+\frac{69 c^{2} \mu^{2}}{338 \lambda^{2}}-\frac{69 c}{169}+\frac{119 \lambda^{2}}{169 \mu^{2}}-\frac{1}{2}$. 
Отметим, что мы получили выражение общего вида для решений уравнения (46), которое не было выведено в работе [21]. Подставив $a_{3}=a_{4}=0, a_{1}=-12 \delta \alpha$, $a_{2}=-12 \delta$ и $z=x-\left(a_{0}+8 \delta \beta+\delta \alpha^{2}\right) t$ в формулы (47)-(49), получим решения уравнения Кортевега-де Фриза, которые были приведены в работе [21].

\section{4. ЗАКЛЮЧЕНИЕ}

Мы доказали, что обобщенное уравнение Буссинеска (4) допускает двухпараметрическую группу симметрий, отвечающую двум группам симметрий. Этот результат показывает, что для уравнения (4) применение метода группы симметрий может порождать только решения типа бегущей волны. Подобные переменные находятся с помощью характеристического уравнения и далее получается приведенная форма исходного нелинейного УЧП, которая представляет собой нелинейное ОДУ. Чтобы получить точные решения, мы применяли две процедуры: прямой метод и метод $\frac{G^{\prime}}{G}$-разложения. С помощью прямого метода для некоторых функций были построены точные решения вида солитонов, кинков, антикинков и компактонов. Применив метод $\frac{G^{\prime}}{G}$-разложения в случае $f(u)=u^{2}$, мы построили три новых типа решений типа бегущей волны.

Благодарности. Работа была частично финансово поддержана DGICYT (проект MTM2006-05031), Junta de Andalucía group FQM-201 и проектом P06-FQM-01448.

\section{Список литературы}

[1] M. J. Boussinesq, C. R. Acad. Sci. Paris, 72 (1871), 755-759.

[2] M. J. Boussinesq, J. Math. Pures Appl., 17 (1872), 55-109.

[3] A. M. Samsonov, E. V. Sokurinskaya, "Energy exchange between nonlinear waves in elastic waveguides and external media", Nonlinear Waves in Active Media, Res. Rep. Phys., ed. J. Engelbrecht, Springer, Berlin, 1989, 99-104.

[4] D. Zhengde, G. Boling, J. Partial Differential Equations, 12:4 (1999), 301-312.

[5] G. Chen, Y. Wang, S. Wang, J. Math. Appl., 299:2 (2004), 563-577.

[6] L. Yacheng, X. Runzhang, J. Math. Anal. Appl., 338:2 (2008), 1169-1187.

[7] D. Zhengde, G. Boling, Acta Math. Sci. B, 20:3 (2000), 322-334.

[8] S. Wang, G. Chen, Nonliniar Anal., 64:1 (2006), 159-173.

[9] P.A. Clarkson, T.J. Priestley, Symmetries of a Generalised Boussinesq equation, http://en. scientificcommons .org/234544.

[10] P. A. Clarkson, J. Phys. A, 22:13 (1989), 2355-2367.

[11] P. A. Clarkson, M. D. Kruskal, J. Math. Phys., 30:10 (1989), 2201-2213.

[12] P. A. Clarkson, D. K. Ludlow, J. Math. Anal. Appl., 186:1 (1994), 132-155.

[13] P. A. Clarkson, Chaos Solitons Fractals, 5:12 (1995), 2261-2301.

[14] M.S. Bruzón, M. L. Gandarias, J. Ramírez, "Similarity reductions of an optical model", Symmetry and Perturbation Theory, eds. G. Gaeta, B. Prinari, S. Rauch-Wojciechowski S. Terracini, World Sci., Singapore, 2005, 59-66.

[15] M.S. Bruzón, M. L. Gandarias, "Applying a new algorithm to derive nonclassical symmetries", Nonlinear Science and Complexity, Proc. Conf. (Beijing, China, 7-12 August 2006), Trans. Nonlinear Sci. and Complexity, 1, eds. A. C. J. Luo, L. Dai, H. R. Hamidzadeh, World Sci., Hackensack, NJ, 2007, 7-12.

[16] M.S. Bruzón, M. L. Gandarias, Commun. Nonlinear Sci. Numer. Simul., 13:3 (2008), $517-523$.

2 Теоретическая и математическая физика, т. 160, № 1, 2009 г. 
[17] M. S. Bruzón, M. L. Gandarias, Proc. Appl. Math. Mech., 8:1 (2009), 10587-10588.

[18] M. L. Gandarias, M. S. Bruzon, J. Nonlinear Math. Phys., 5:1 (1998), 8-12.

[19] Н. Х. Ибрагимов, Группь преобразований в математической физике, Наука, М., 1983.

[20] П. Олвер, Приложения групп Ли к дифференииальным уравнениям, Мир, М., 1989.

[21] M. Wang, X. Li, J. Zhang, Phys. Lett. A, 372:4 (2008), 417-423.

[22] М. Абрамовиц, И. Стегун (ред.), Справочник по специалъным функииям с формулами, графиками и математическими таблицами, Наука, М., 1979.

[23] J. C. Camacho, M.S. Bruzón, J. Ramírez, M. L. Gandarias, "Local symmetries and exact solutions of a beam equation", Chaos Solitons Fractals, to be published. 